\title{
Medical examinations for pilots
}

\author{
PJ Nicholson
}

\section{Fitness for work}

\section{Summary}

High altitude is an environment that is alien to man. Stressors associated with the mechanics of flight include motion and acceleration forces applied in three vectors and in degrees that are foreign to the human vestibular apparatus. Furthermore, the work patterns of a pilot can interfere with lifestyle and circadian rhythms. Therefore medical fitness is an important consideration in determining an individual's suitability to exercise a pilot's licence. The medical standards applied depend on the type of aircraft flown and the duties expected of a pilot. There are three broad categories of pilot. In ascending order of stringency of medical standards these are the private pilot, the professional pilot and the military pilot.

Keywords: pilots, medical examinations, fitness for work

\section{Determination of fitness for} work

- effects of health on work

- effects of work on health

Box 1

\section{Hazardsof altitude}

- reduced atmospheric pressure

- reduced partial pressure of oxygen

- reduced temperature

Box 2

\footnotetext{
7 Hadrian Court, Darlington DL3 8RE, UK

PJ Nicholson
}

Accepted 26 April 1995
The main purpose of any medical examination that determines fitness for work is to ensure that an individual is fit to perform the task without risk to the health and safety of that individual and others (box 1). An individual should not be ascribed work that he or she is not capable of performing effectively or safely by virtue of some medical condition. Similarly an individual should not be allowed to perform work where the nature of that work could aggravate an underlying medical condition.

In order to make a sound decision regarding an individual's fitness for work, the medical examiner must understand all relevant work factors. These work factors include the nature of the hazards in the working environment, the risk and degree of exposure to these hazards, the protective equipment available and organisational factors such as work patterns.

\section{The aviation environment}

\section{ALTITUDE}

The physiological stresses of aviation are principally those associated with working at high altitude (box 2). Within the innermost layer of the atmosphere, that extends from sea level to 25000 to 60000 feet (depending on latitude and season), temperature falls by $2^{\circ} \mathrm{C}$ for every 1000 feet climbed. ${ }^{1}$ Atmospheric pressure at a given altitude is the result of a combination of gravitational force and compression by the mass of air above it. Atmospheric pressure at sea level is $760 \mathrm{mmHg}$ and the fall in atmospheric pressure with increasing altitude is almost exponential (figure).

Oxygen constitutes $21 \%$ of dry air and the partial pressure of oxygen in air falls in the same manner as atmospheric pressure. However, the sigmoid shape of the oxygen dissociation curve produces a built-in protection against hypoxia. At 10000 feet, atmospheric pressure falls by over $30 \%$, however, haemoglobin is $90 \%$ saturated with oxygen. On further ascent saturation falls rapidly to produce overt signs and symptoms of hypoxia.

At constant temperature, the volume of a gas is inversely related to the pressure exerted upon it (Boyle's law). As atmospheric pressure falls, so gas contained in body cavities will expand. On descent gas will contract as cabin pressure increases. Changes in gas volume effect various body systems (see box 3).

Cabin pressurisation protects the occupants of an aircraft against the effects of altitude, ie, hypoxia and decompression sickness. Although a commercial passenger-carrying aircraft may cruise at about 35000 feet, where atmospheric pressure is $179 \mathrm{mmHg}$, the cabin is pressurised to an equivalent altitude of 5000-6000 feet. At these cabin altitudes, atmospheric pressure is 632 and $609 \mathrm{mmHg}$, respectively.

Pressurised cabins and pressurisation equipment place penalties on aircraft performance, by virtue of added weight and by using engine power. Therefore in high-performance combat aircraft the pilot is protected from the effects of altitude differently. ${ }^{2}$ The cabin is pressurised to a lesser extent and the pilot breathes oxygen-enriched air through personal breathing apparatus. By maintaining a low pressure differential between the cockpit and the environment, the risk of decompression sickness is reduced. There is an increased likelihood of sudden decompression in military aircraft because of potential enemy action. Cockpit pressurisation and oxygen enrichment of the personal air supply begin at altitudes above 5000 feet. The equivalent altitude within the cockpit is maintained at about half actual altitude.

At cockpit altitudes above 40000 feet, however, even the pressure of $100^{\circ}$ o oxygen is not sufficient to oxygenate the blood adequately. The oxygen supply to the mask is then pressurised. Pressure breathing is only used for short periods since the rise in intrathoracic pressure affects the dynamics of the cardiovascular and respiratory systems and the procedure is tiring. 


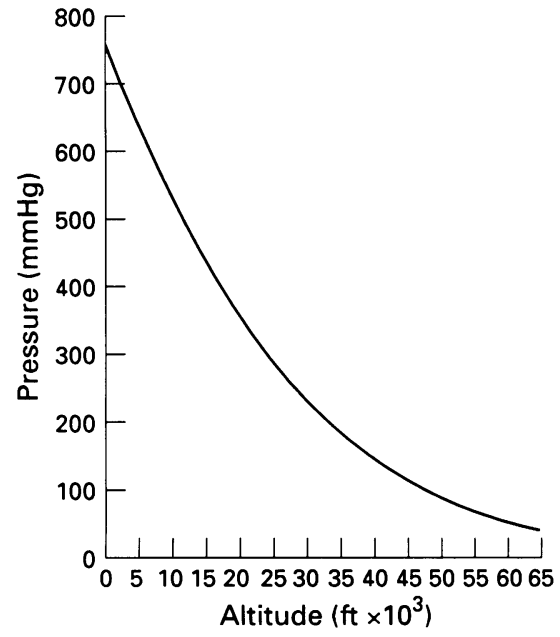

Figure Relationship between altitude and pressure

\begin{tabular}{|l|}
\hline $\begin{array}{l}\text { Body systems affected by } \\
\text { change in gas volume }\end{array}$ \\
\hline - middle ear \\
- paranasal sinuses \\
- gastrointestinal tract \\
- lungs \\
\hline
\end{tabular}

Box 3
MOTION

Fully trained professional pilots rarely suffer from motion sickness, but it may be a problem in other aircrew such as navigators, cabin crew and trainee pilots. Individuals who may have ongoing problems are those who are highly receptive to the sensory stimuli of motion, who adapt slowly on repeated exposure and who do not retain adaptation to motion. The extent of symptoms will determine whether they can continue flying and in what type of aircraft (manoeuvres in combat aircraft are more likely to give rise to provocative stimuli).

When flying under conditions of good visibility, the pilot can perceive accurately his or her orientation in space. Visual references during flight can be hampered by flying at night or in bad weather. At such times, the aircraft is flown on the basis of information obtained from instruments and a greater reliance on special senses other than vision. This may give rise to disorientation in relation to the position, attitude or movement of the aircraft, ie, spatial disorientation. Spatial disorientation can also be a consequence of unusual visual cues (eg, the pilot may assume that a sloping cloud top is horizontal since experience tells him that most cloud tops are horizontal) or by acceleration forces affecting the vestibular apparatus. Acceleration forces may be linear or radial and experienced in three vectors, ie, fore-aft, transversely or vertically.

Military pilots who fly high-performance combat aircraft are also subject to long-duration acceleration (ie, greater than 2 seconds). This is measured as $G$, $1 \mathrm{G}$ being equivalent to the force of gravity. Radial acceleration acting through the long axis of the body has the greatest effect on the body and human performance. Headward acceleration as a result of centrifugal force produces a footward inertial response, thereby impeding venous return from the lower limbs. Cardiac output is reduced and retinal and cerebral perfusion is impaired. Tunnel vision, loss of vision and ultimately G-induced loss of consciousness (G-LOC) may occur. ${ }^{3}$

\section{Work organisation}

In combat aircraft, a pilot may fly in a single-seat aircraft, or as the only pilot in a two-seat aircraft, flying with a navigator. In larger aircraft, the professional pilot will be part of a team. That team comprise two pilots, ie, a captain and co-pilot and other front crew, such as navigator, flight engineer, etc. Depending on the sophistication of the aircraft and the circumstances of the flight the pilot may fly 'hands on'. However, there is an increasing trend towards automation and a move from conventional instruments to computerised visual display units. In most modern airliners the role of the pilot has become much more that of a systems operator than the traditional hands-on aviator.

The work of the long-haul airline pilot is such that it may involve frequent long journeys often at inconvenient times. This can affect lifestyle, such as patterns of diet and sleep. Flying at night or transmeridian flight that involves crossing three or more time zones interferes with circadian rhythms and may cause desynchronisation or 'jet lag'. Desynchronisation affects both physiological and psychological rhythms and produces physical symptoms and impairment of psychomotor performance.

As with any traveller who visits tropical areas, the pilot will run the risk of acquiring exotic infections and there will be a need for appropriate prophylactic procedures such as immunisation and anti-malarial medication.

\section{The job}

Whether the role of an aircraft is to transport passengers, cargo or weapons the common need is for the pilot to fly the aircraft safely. Aircraft are an expensive mode of transport that travel in an environment that is foreign to man. Pilots require special skills including an ability to work effectively at times of high workload. In multi-crew aircraft the pilot must be able to work effectively with others.

An aircraft, by virtue of its size, weight and the amount of fuel carried is a potentially hazardous object. An accident involving the loss of an aircraft will have serious consequences for passengers and for the general public and the environment under the flight path. Flight safety and the safety of the general public dictate that professional pilots satisfy the highest standards. Applicants are therefore selected for training as a pilot on the basis of academic achievement, personality, technical aptitude, and medical fitness.

The cost of training a professional pilot is high and this influences recruitment policies. A prospective employer, be it an airline or the military, will consider not only the current medical fitness of an applicant, but also the likelihood of an applicant remaining fit to fly throughout his or her working life. ${ }^{4}$ 


\begin{tabular}{|l|}
\hline Medical assessment for \\
pilots \\
\hline - height, weight \\
- blood pressure, urinalysis \\
- visual acuity, convergence, visual \\
fields, fundoscopy \\
- otoscopy, hearing \\
- palpultation of the chest \\
- hernial orifices abdomen and \\
- assessment of range of movements \\
- reflexes and joints \\
\hline
\end{tabular}

Box 4

\section{Medical conditions \\ incompatible with the duties of a pilot}

- cardiovascular: myocardial infarction, angina, hypertension, aortic or mitral stenosis, cardiomyopathy

- respiratory: asthma, pneumothorax, bullous disease, sarcoidosis, emphysema, chronic bronchitis, active tuberculosis

- haematological: anaemia, sickle cell trait

- gastrointestinal: peptic ulcer, inflammatory bowel disease, chronic pancreatitis

- endocrine: diabetes mellitus, familial hyperlipidaemia

- urogenital: renal calculi, polycystic kidneys, chronic renal failure, renal transplantation, pregnancy

- neurological: epilepsy, migraine, recent head injury disturbance of consciousness, multiple sclerosis

- psychiatric: psychosis, alcohol or drug dependence, personality disorder, significant neurosis

- ear, nose and throat: otitis media, deafness, vestibular disorders

- eyes: glaucoma, cataract, amblyopia, keratitis

- miscellaneous: obesity, malignancy

Box 5

\section{Medical standards}

Having an awareness of the varied roles of pilots, one may appreciate that the medical standards applied depend upon the type of flying licence held. The required medical standards are higher for a military pilot than for a professional pilot because of the greater range of environmental hazards, the more severe degree and the greater likelihood of exposure. Similarly, the medical standards applied to a professional pilot, who is regularly in charge of a large aircraft for many hours at a time are higher than those for a private pilot who flies a light aircraft for an hour or so on occasional weekends.

The minimum medical standards for fitness to fly as a pilot are advised by the International Civil Aviation Organisation, which is an agency of the United Nations. These standards are applied in each country by national regulatory authorities. Some variation does exist between countries, eg, in the periodicity of examinations. Because of European legislation, all European Community member states will harmonise medical standards in 1996.

International standards $s^{5}$ are phrased in general terms, with the exception of critical areas, eg, vision (table). The basic rule in determining fitness to fly is that the applicant for a medical certificate shall be free from:

- any abnormality, congenital or acquired, or

- any active, latent, acute or chronic disability, or

- any wound, injury or sequelae of surgery,

that is likely to interfere with the safe operation of an aircraft or the safe performance of duties.

\section{The examination}

In the UK, the initial medical examination for professional licences is performed at the medical department of the Civil Aviation Authority. This medical department is also responsible for appointing authorised medical examiners at locations around the country. These examiners are either licensed to perform examinations on student and private pilots only or they are licensed to perform examinations on all classes of aircrew. This includes renewal examinations on professional pilots, navigators, flight engineers and air traffic control officers. In the UK, examiners will have undertaken a training course in aviation medicine before being appointed.

The frequency of medical examinations and the content of the examination is determined by the type of licence held and the age of the licence holder.

Prior to the examination, the pilot completes a questionnaire and signs a health declaration. The initiation examination of applicants includes an Ishihara test for colour perception. If the application fails this test, then a Holmes-Wright lantern is used to establish perception of signal red, signal green and white, ie, colours of lights used on airfields for safety purposes.

Renewal medical examinations are otherwise the same as the initial assessment and include the items shown in box 4. Depending on the type of licence held and the age of the pilot an electrocardiogram and audiometric tests may also be performed.

\section{Is the pilot fit to fly?}

Some medical conditions are not compatible with the issue of a medical certificate of fitness (box 5). Other medical conditions will require specialist opinion and particularly in the case of professional aircrew, decision by the regulatory authority. The more hazardous nature of military flying (pressure breathing, risk of decompression, $\mathrm{G}$ forces, ejection seats, ability to survive in foreign climates, etc) dictate higher standards.

Table Visual standards for professional and private pilots

\begin{tabular}{lll}
\hline & \multicolumn{1}{l}{ Licence } & \\
\cline { 2 - 3 } & Professional & \multicolumn{1}{c}{ Private } \\
\hline Distance vision & $6 / 96 / 9$ (corrected) & $6 / 126 / 12$ (corrected) \\
$\begin{array}{l}\text { Near vision } \\
\begin{array}{l}\text { Middle vision } \\
\text { Correction }\end{array}\end{array}$ & $\begin{array}{l}\mathrm{N} 5 \text { at } 30-50 \mathrm{~cm} \\
\mathrm{~N} 14 \text { at } 100 \mathrm{~cm} \\
\text { not to exceed } \pm 3 \mathrm{D}\end{array}$ & $\mathrm{N} 5$ at $30-50 \mathrm{~cm}$ \\
& & not to exceed $\pm 5 \mathrm{D}$
\end{tabular}


A pilot may or may not be allowed to fly depending on the medical condition and whether the pilot is an initial applicant for a licence or a qualified pilot. For some conditions a past history will prevent a person from flying, eg, psychosis, drug abuse, epilepsy. For other medical conditions, it is active disease rather than a past history that will preclude flying, eg, peptic ulceration.

A trained and experienced professional pilot may be allowed to continue flying with a condition that would preclude recruitment. A typical example is monocularity. ${ }^{6}$ The trained pilot is familiar with the aviation environment and its visual cues and a restricted licence will be permitted, ie, fit to fly 'as or with co-pilot'.

The commonest causes of loss of licence in professional aircrew are cardiovascular disease, neuropsychiatric illness, and malignancy. ${ }^{7}$ Pregnancy will prevent a female pilot from flying, although she may return to flying duties during the second trimester and with the restriction 'as or with co-pilot'.

\section{Medication}

Often it is not the drug taken that is the limiting factor in determining fitness to fly, but rather it is the underlying medical condition. For example, a pilot who takes indomethacin is likely to have such a degree of joint problems that they alone will preclude flying. Also, a pilot should not fly whilst he has an acute infection and therefore guidelines regarding antibiotics are usually unnecessary.

Drugs that have central effects or those that affect vision are not compatible with continuing flying duties. Among these are anticholinergics, psychotropics and antihistamines. Terfenadine is safe for use by pilots. However, it remains wise to establish by a period of use on the ground that there are no idiosyncratic reactions before allowing the pilot to fly. Drugs that cause significant biochemical changes, eg, oral steroids are also contraindicated.

The use of cromoglycate and inhaled steroids for mild asthma is compatible with continued civilian flying. ${ }^{8}$ The occasional use of inhaled salbutamol may also be acceptable, provided that it does not cause tremor. If the severity of asthma is such that there are more frequent episodes, then the illness itself and the drugs required for control (oral salbutamol, theophylline) will terminate a pilot's career.

In hypertension, provided that there is good control and that there is no end organ damage, the following drugs are permissible - thiazide diuretics, atenolol, lisinopril, captopril, enalapril. ${ }^{7}$ Other anti hypertensive medication, including propranolol, is not permitted.

In diabetes mellitus, biguanides rarely cause hypoglycaemia and this class of drug is compatible with restricted flying..$^{9}$ Sulphonylureas and insulin are not compatible with the safe exercise of a flying licence.

1 Ernsting J. The aerospace environment and its effect on the lungs. In: Parkes WR, ed. Occupational lung disorders. 3rd edn. Oxford: Butterworth-Heinemann, 1994.

2 Macmillan AJF. The pressure cabin. In: Ernsting J, King P, eds. Aviation medicine. 2nd edn. sting J, King P, eds. Aviation med

3 Glaister DH. The effects of long duration acceleration. In: Ernsting J, King P, eds. Aviaacceleration. In: Ernsting J, King P, eds. Aviation medi 1988.

4 Bennett G. Civil Aviation. In: Cox RAF, Edwards FC, McCallum RI, eds. Fitness for work, 2nd edn. Oxford: Oxford Medical Publications, 1995.
5 International Civil Aviation Organisation. International standards and recommended practices. Personnel licensing. Annex 1. 8th edn. Montreal: ICAO, 1988.

6 Cloherty J. Ophthalmological conditions and the examination of the eye. In: Ernsting J, King $\mathrm{P}$, eds. Aviation medicine. 2nd edn. London: Butterworths, 1988 .

7 Civil Aviation Authority (CAA). Guidance notes for authorised medical examiners. London: CAA, 1993.
8 Hopkirk JAC. Flying with existing respiratory disease. In: Parkes WR, ed. Occupational lung disorders. 3rd edn. Oxford: ButterworthHeinemann, 1994.

9 Hull DH. Other important medical conditions. In: Ernsting J, King P, eds. Aviation medicine. 2nd edn. London: Butterworths, 1988. 